\title{
Some exact results for a trapped quantum gas at finite temperature
}

\author{
Brandon P. van Zyl, Rajat K. Bhaduri \\ Department of Physics and Astronomy, McMaster University, Hamilton, Ontario, Canada, L8S 4 M1 \\ Akira Suzuki \\ Tokyo University of Science, Kagurazaka 1-3, Shinjuku-ku, Tokyo 162-8601, Japan \\ Matthias Brack \\ Institut fü̈ Theoretische Physik, Universität Regensburg, D-93040 Regensburg, Germany
}

\begin{abstract}
We present closed analytical expressions for the particle and kinetic energy spatial densities at finite temperatures for a system of noninteracting fermions (bosons) trapped in a $d$-dimensional harmonic oscillator potential. For $d=2$ and 3 , exact expressions for the $N$-particle densities are used to calculate perturbatively the temperature dependence of the splittings of the energy levels in a given shell due to a very weak interparticle interaction in a dilute Fermi gas. In two dimensions, we obtain analytically the surprising result that the $l$-degeneracy in a harmonic oscillator shell is not lifted in the lowest order even when the exact, rather than the Thomas-Fermi expression for the particle density is used. We also demonstrate rigorously (in two dimensions) the reduction of the exact zero-temperature fermionic expressions to the Thomas-Fermi form in the large- $N$ limit.
\end{abstract}

PACS numbers: 03.75.-b, 03.75.Fi, 05.30.Fk

\section{INTRODUCTION}

The observation of Bose-Einstein condensation in ultra-low temperature trapped atomic gases (see e.g., Ref. [1] for a comprehensive review), along with the recent experimental effort of Jin and DeMarco [2] in achieving a degenerate Fermi gas, has sparked a renewed interest in the study of the thermodynamic properties of trapped quantum gases. For a dilute gas of trapped bosons or fermions, assuming the interparticle interactions to be very weak, the manybody system may be modeled in the zeroth-order approximation as a system of noninteracting particles. This is an attractive situation from a theoretical point of view because the thermodynamics of the noninteracting $N$-body system can be calculated from the single-particle partition function of the associated trapping potential. For the case of Bose statistics, exact closed form expressions for the thermodynamic quantities of the ideal trapped gas can be obtained at all temperatures in any dimension provided the trapping potential is an harmonic oscillator (HO). An ideal trapped gas of fermions on the other hand is not as easy to analyze. Unlike the Bose gas, the Fermi distribution function cannot be expanded as a power series for all temperatures, and as a result, closed form expressions for e.g., the particle and kinetic energy densities, at finite temperature are difficult to obtain. Indeed, it is only recently that Brack and van Zyl [3] have constructed exact, analytical expressions for the particle and kinetic energy densities of a harmonically confined ideal Fermi gas in any spatial dimension. These expressions are useful for both numerical and analytical computations, but are restricted to zero temperature. Prior to the work in Ref. [3], fermions in harmonic traps have been studied using exact numerical [4, 5, 6, 7, analytical [8, 9, 10] and semiclassical techniques [11]. The exact analytical results for the particle and kinetic energy densities, however, are limited to one dimension (1D) at zero temperature [9] or arbitrary dimensions in the high temperature regime 10, 12].

In this article, our main emphasis will be on presenting exact analytical results for an ideal $d$-dimensional trapped Fermi gas at any finite temperature, thereby extending the work in Ref. [3] beyond $T=0$. We shall also digress to give some novel analogous expressions for the ideal trapped Bose gas which have not yet appeared in the literature. The trapping potential will be taken to be an isotropic $\mathrm{HO}$, although our results are easily generalized to the anisotropic case. Apart from the intrinsic merit of exact results for a many-body system, our findings should also prove useful in the density-functional theory of weakly interacting inhomogeneous Fermi systems.

The rest of our paper is organized as follows: In Sec. II, we derive analytical expressions for the finite-temperature particle and kinetic energy densities for an ideal trapped Fermi gas in arbitrary dimensions. We also show rigorously how the exact quantum expressions at $T=0$ asymptotically approach their Thomas-Fermi (TF) results in the large- $N$ limit; we know of only two other examples where such a result has been rigorously established analytically [9, 13. Then, in Sec. III, we derive expressions analogous to the fermionic system for a trapped gas of noninteracting bosons. In Sec. IV, we apply our exact result for the spatial density to calculate the mean-field potential, and the resulting finitetemperature splitting of the energy levels in a $d=3 \mathrm{HO}$ shell perturbatively. Although a similar zero temperature calculation has already been considered numerically by Heiselberg and Mottelson 14], our results (in contrast to Ref. [14]) do not rely on the TF approximation for the particle density. The corresponding calculation for $d=2$ yields 
an interesting result, which is to be discussed in Sec. IV B. Finally, in Sec. V, we summarize our results and suggest other possible avenues for future research.

\section{IDEAL FERMI GAS AT FINITE TEMPERATURE}

\section{A. Single-particle density in $d$ dimensions}

The starting point in our investigation is a system of noninteracting fermions at zero temperature described by the time-independent Schrödinger equation

$$
\hat{H} \phi_{i}(\mathbf{r})=[\hat{T}+V(\mathbf{r})] \phi_{i}(\mathbf{r})=\varepsilon_{i} \phi_{i}(\mathbf{r})
$$

where $V(\mathbf{r})$ is a one-body potential to be specified later (all $\epsilon_{i}$ 's are taken to be positive). The single-particle density matrix can be obtained by an inverse Laplace transform of the zero-temperature Bloch density matrix, $C_{0}\left(\mathbf{r}, \mathbf{r}^{\prime}\right)$ :

$$
\rho\left(\mathbf{r}, \mathbf{r}^{\prime}\right)=2 \sum_{\varepsilon_{i}<E_{f}} \phi_{i}^{\star}\left(\mathbf{r}^{\prime}\right) \phi_{i}(\mathbf{r}) \Theta\left(E_{f}\right)=\mathcal{L}_{E_{f}}^{-1}\left[\frac{2}{\beta} C_{0}\left(\mathbf{r}, \mathbf{r}^{\prime} ; \beta\right)\right]
$$

where

$$
C_{0}\left(\mathbf{r}, \mathbf{r}^{\prime} ; \beta\right)=\sum_{\text {all } i} \phi_{i}^{\star}\left(\mathbf{r}^{\prime}\right) \phi_{i}(\mathbf{r}) \exp \left(-\beta \varepsilon_{i}\right)
$$

and $E_{f}$ is the Fermi energy; the factor of two accounts for spin. We have put in the unit step-function $\Theta\left(E_{f}\right)$ in Eq. (2) so that the Laplace transform with respect to $E_{f}$ may formally be taken to be two-sided [15. Note that in quantum statistical mechanics, $\beta$ is usually identified with the inverse temperature, $\beta=1 / k_{B} T$. However, in our present context, $\beta$ is to be interpreted as mathematical variable which in general is taken to be complex, and not the inverse temperature $1 / k_{B} T$.

At finite-temperature, the single-particle density matrix is obtained from the Bloch density matrix by using the relation 16]

$$
\rho\left(\mathbf{r}, \mathbf{r}^{\prime} ; T\right)=\mathcal{L}_{\mu}^{-1}\left[\frac{2}{\beta} C_{T}\left(\mathbf{r}, \mathbf{r}^{\prime} ; \beta\right)\right]
$$

where

$$
C_{T}\left(\mathbf{r}, \mathbf{r}^{\prime} ; \beta\right)=C_{0}\left(\mathbf{r}, \mathbf{r}^{\prime} ; \beta\right) \frac{\pi \beta T}{\sin (\pi \beta T)},
$$

is the finite-temperature Bloch density matrix, and $\mu$ is the chemical potential. In Eq. (4), the Laplace transform with respect to $\mu$ is two-sided, so that $\mu$ is allowed to go negative. Specializing now to the case of an isotropic harmonic oscillator in $d$ dimensions, viz.,

$$
V(\mathbf{r})=\frac{1}{2} m \omega_{0}^{2} r^{2}, \quad r=\sqrt{x_{1}^{2}+x_{2}^{2}+\cdots+x_{d}^{2}},
$$

we have for the zero-temperature Bloch density matrix 17

$$
C_{0}^{(d)}\left(\mathbf{r}, \mathbf{r}^{\prime} ; \beta\right)=C_{0}^{(d)}(q, s ; \beta)=\left(\frac{1}{2 \pi}\right)^{d / 2} \frac{1}{\sinh ^{d / 2}(\beta)} \exp \left\{-\left[q^{2} \tanh (\beta / 2)+\left(s^{2} / 4\right) \operatorname{coth}(\beta / 2)\right]\right\}
$$

In the above expression (and what follows), all lengths and energies have been scaled by $l_{0}=\sqrt{\hbar / m \omega_{0}}$ and $\hbar \omega_{0}$, respectively, and we have introduced the center-of-mass and relative coordinates:

$$
\mathbf{q}=\frac{1}{2}\left(\mathbf{r}+\mathbf{r}^{\prime}\right), \quad \mathbf{s}=\mathbf{r}-\mathbf{r}^{\prime}
$$

In order to obtain the finite-temperature single-particle density, we need to set $s=0$ in Eq. (7) and along with Eq. (5), perform the inverse Laplace transform given by Eq. (4). This is a difficult task if one attempts to use Eq. (7) in its present form. However, matters can be simplified considerably if one uses the following identity [3, 18],

$$
\exp \{-x \tanh (\beta / 2)\}=\sum_{n=0}^{\infty}(-1)^{n} L_{n}(2 x) e^{-x}\left\{e^{-n \beta}+e^{-(n+1) \beta}\right\}
$$


in Eq. (7), which for $s=0$ now reads

$$
C_{0}^{(d)}(q ; \beta)=\left(\frac{1}{2 \pi}\right)^{d / 2} \frac{1}{\sinh ^{d / 2}(\beta)} \sum_{n=0}^{\infty}(-1)^{n} L_{n}\left(2 q^{2}\right) e^{-q^{2}}\left\{e^{-n \beta}+e^{-(n+1) \beta}\right\} .
$$

Substituting Eq. (10) into Eq. (5) and performing the inverse Laplace transform, Eq. (4), leads to the finitetemperature single-particle density in any dimension. In order to illustrate the method, we will now proceed to give an explicit calculation for the case of two dimensions (2D), followed by a statement of the general result in arbitrary dimensions.

\section{Two dimensions}

The 2D system turns out to be the simplest case of all, and for this reason, we give here a detailed derivation of the finite-temperature single-particle density. We begin by noting the following important exact inverse Laplace transforms (all two-sided):

$$
\begin{gathered}
\mathcal{L}_{\eta}^{-1}\left[\frac{e^{-n \beta}}{\sinh (\beta)}\right]=2 \sum_{k=0}^{\infty} \delta(\eta-(2 k+1)-n) \Theta(\eta), \\
\mathcal{L}_{\mu}^{-1}\left[\frac{\pi T}{\sin (\pi \beta T)}\right]=\frac{1}{\left[\exp \left(-\frac{\mu}{T}\right)+1\right]} .
\end{gathered}
$$

Putting $d=2$ in Eq. (10) and using Eqs. (4), (5), the finite-temperature density is given by

$$
\rho^{(2)}(\mathbf{q} ; T)=\left(\frac{1}{\pi}\right) \sum_{n=0}^{\infty}(-1)^{n} L_{n}\left(2 q^{2}\right) e^{-q^{2}} \times \mathcal{L}_{\mu}^{-1}\left[\left(\frac{e^{-n \beta}+e^{-(n+1) \beta}}{\sinh (\beta)}\right) \frac{\pi T}{\sin (\pi \beta T)}\right] .
$$

Applying the convolution theorem for Laplace transforms [15] and making use of Eqs. (11), (12), we immediately obtain

$$
\begin{aligned}
\rho^{(2)}(\mathbf{q} ; T) & =\left(\frac{2}{\pi}\right) \sum_{n=0}^{\infty}(-1)^{n} L_{n}\left(2 q^{2}\right) e^{-q^{2}} \times \sum_{k=0}^{\infty}\left\{\int_{-\infty}^{\infty} d \tau \delta(\tau-(2 k+1)-n) \frac{1}{\left[\exp \left(\frac{\tau-\mu}{T}\right)+1\right]}\right. \\
& \left.+\int_{-\infty}^{\infty} d \tau \delta(\tau-(2 k+2)-n) \frac{1}{\left[\exp \left(\frac{\tau-\mu}{T}\right)+1\right]}\right\} \\
& =\left(\frac{2}{\pi}\right) \sum_{n=0}^{\infty}(-1)^{n} L_{n}\left(2 q^{2}\right) e^{-q^{2}} \times \sum_{k=0}^{\infty}\left\{\frac{1}{\left[\exp \left(\frac{2 k+n+1-\mu}{T}\right)+1\right]}+\frac{1}{\left[\exp \left(\frac{2 k+n+2-\mu}{T}\right)+1\right]}\right\} \\
& =\left(\frac{2}{\pi}\right) \sum_{n=0}^{\infty}(-1)^{n} L_{n}\left(2 q^{2}\right) e^{-q^{2}} \times \sum_{k=0}^{\infty} \frac{1}{\left[\exp \left(\frac{\varepsilon_{n}+k-\mu}{T}\right)+1\right]} \\
& =\left(\frac{2}{\pi}\right) \sum_{n=0}^{\infty}(-1)^{n} L_{n}\left(2 q^{2}\right) e^{-q^{2}} F_{n}^{(2)}(\mu)
\end{aligned}
$$

where the function $F_{n}^{(2)}(\mu)$ is defined as

$$
F_{n}^{(2)}(\mu)=\sum_{k=0}^{\infty} \frac{1}{\left[\exp \left(\frac{\varepsilon_{n}+k-\mu}{T}\right)+1\right]}
$$

and $\varepsilon_{n}=(n+1)$ is the noninteracting energy spectrum (in scaled units) of the 2D harmonic oscillator potential. In the limit $T \rightarrow 0$, the Fermi function in Eq. (15) goes over to the Heaviside step function, and when filling $M+1$ oscillator shells, Eq. (14) reduces to

$$
\rho^{(2)}(q)=\left(\frac{2}{\pi}\right) \sum_{n=0}^{M}(M-n+1)(-1)^{n} L_{n}\left(2 q^{2}\right) e^{-q^{2}} .
$$




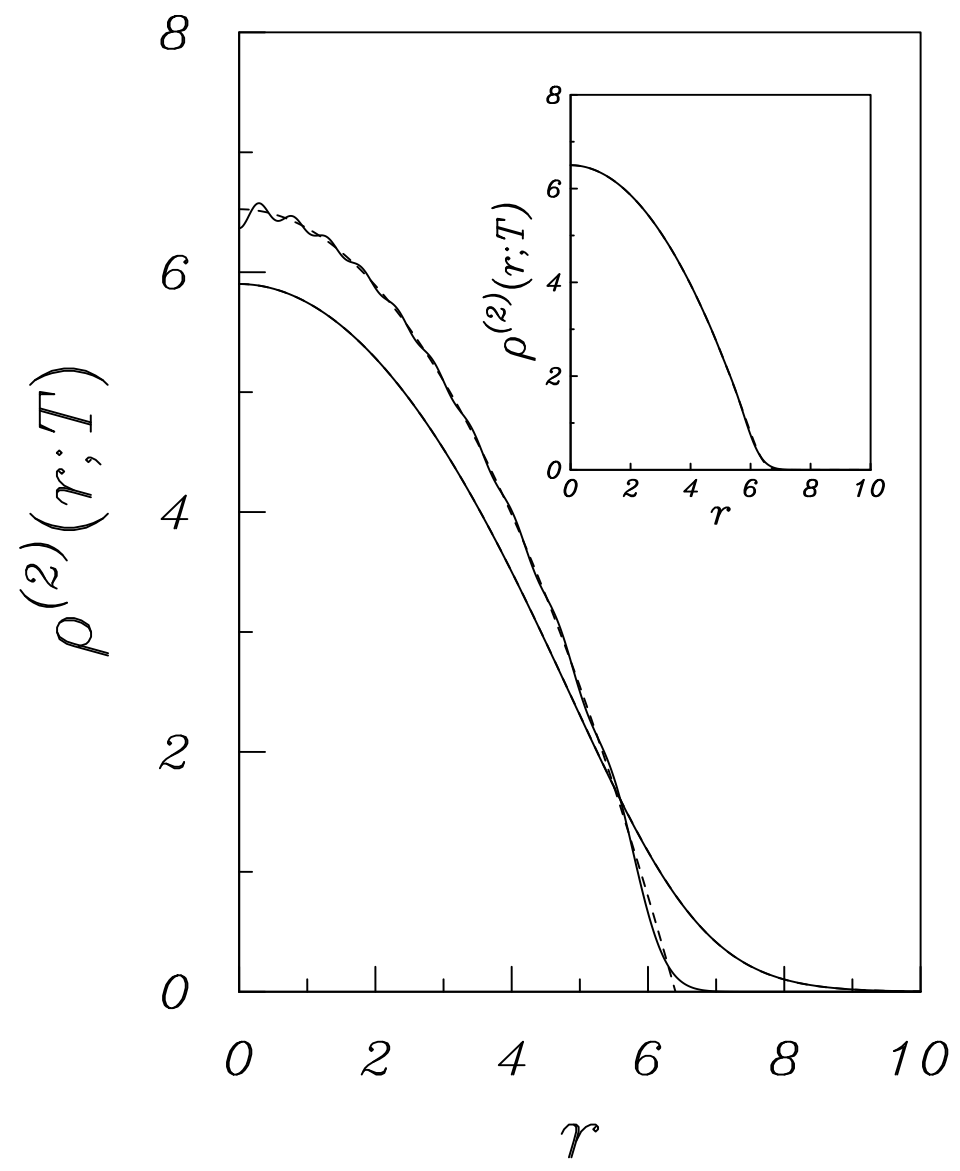

FIG. 1: The 2D exact (solid curves) and TF (dashed curves) number densities at various temperatures for $N=420$ particles. The upper curves in the main figure correspond to $T=0$, while the lower-lying curves are for $T=5$. The figure inset shows the $2 \mathrm{D}$ exact and $\mathrm{TF}$ densities at $T=1$. All quantities have been scaled as discussed in the text.

Equation (16) is of course identical to the result obtained in Ref. [3] in which only zero-temperature quantities were investigated. In Fig. 1, we display the exact 2D radial density (solid curves), $\rho^{(2)}(r ; T)$, for $N=420$, where the lowest 20 shells are filled. For comparison, we have also displayed the corresponding 2D TF densities (dashed curves) at zero [3] and finite temperatures [19]. We have used Eqs. (14) and (16) for the exact nonzero and zero-temperature density respectively. In all plots, $T$ denotes the dimensionless quantity $\left(\frac{k_{B} T}{\hbar \omega}\right)$. We note from Fig. 1 that the oscillations due to shell effects are already washed out by $T=1$, resulting in TF and exact densities being almost indistinguishable.

In the high temperature regime where $\mu<\varepsilon_{0}$, we can make use of the expansion

$$
\frac{1}{\left[\exp \left(\frac{2 k+\varepsilon_{n}-\mu}{T}\right)+1\right]}=\sum_{j=1}^{\infty}(-1)^{j+1} e^{j \mu / T} e^{-j(n+1) / T} e^{-2 j k / T}
$$

in Eq. (14). The $k$ sums can be evaluated exactly, and we obtain

$$
\rho^{(2)}(\mathbf{q} ; T)=2 \sum_{j=1}^{\infty}(-1)^{j+1} e^{j \mu / T} C_{0}^{(2)}(\mathbf{q} ; j / T)
$$

Making the formal identification of $\beta=1 / T$, Eq. (18) can be re-written as

$$
\rho^{(2)}(\mathbf{q} ; T)=2 \sum_{j=1}^{\infty}(-1)^{j+1} e^{j \beta \mu} C_{0}^{(2)}(\mathbf{q} ; j \beta) .
$$


A result identical to Eq. (19) has recently been obtained by Wang [10], but as we have discussed above, this form for the density is only valid for $\mu<\varepsilon_{0}$. In contrast, our Eq. (14) is valid at all temperatures and appears in a form that clearly displays the role of the quantum statistics of the system.

Finally, an interesting relationship between $F_{n}^{(2)}(\mu)$ and the Fermi distribution function can be obtained through the normalization condition

$$
\begin{aligned}
N & =\int d^{2} q \rho^{(2)}(\mathbf{q} ; T) \\
& =2 \sum_{n=0}^{\infty} F_{n}^{(2)}(\mu) \\
& =2 \sum_{n=0}^{\infty} \frac{(n+1)}{\left[\exp \left(\frac{\varepsilon_{n}-\mu}{T}\right)+1\right]}
\end{aligned}
$$

\section{Arbitrary dimensions}

The generalization of the above analysis to arbitrary dimensions is straightforward. First, since we are only interested in the diagonal Bloch density matrix, we can immediately set $s=0$ in Eq. (7). Furthermore, we observe that in any dimension

$$
\sinh ^{(1-d / 2)}(\beta)=2^{(d / 2-1)} e^{\beta(1-d / 2)}\left(1+\sum_{m=1}^{\infty} g_{m}^{(d)} e^{-2 m \beta}\right)
$$

Multiplying the numerator and denominator of Eq. (7) by Eq. (21), and using the identity, Eq. (9), we obtain

$$
C_{0}^{(d)}(\mathbf{q} ; \beta)=\frac{1}{\pi^{d / 2}} \frac{1}{\sinh (\beta)} \sum_{n=0}^{\infty}(-1)^{n} L_{n}\left(2 q^{2}\right) e^{-q^{2}}\left(e^{-(n+d / 2-1) \beta}+e^{-(n+d / 2) \beta}\right)\left(1+\sum_{m=1}^{\infty} g_{m}^{(d)} e^{-2 m \beta}\right)
$$

Proceeding exactly as in the 2D case, we finally obtain in any dimension

$$
\rho^{(d)}(\mathbf{q} ; T)=2\left(\frac{1}{\pi}\right)^{d / 2} \sum_{n=0}^{\infty} F_{n}^{(d)}(\mu)(-1)^{n} L_{n}\left(2 q^{2}\right) e^{-q^{2}}
$$

where

$$
F_{n}^{(d)}(\mu)=\sum_{k=0}^{\infty}\left(\frac{1}{\exp \left[\left(\varepsilon_{n}^{(d)}+k-\mu\right) / T\right]+1}+\sum_{m=1}^{\infty} \frac{g_{m}^{(d)}}{\exp \left[\left(\varepsilon_{n}^{(d)}+k+2 m-\mu\right) / T\right]+1}\right)
$$

and $\varepsilon_{n}^{(d)}=n+d / 2$. The expansion coefficients for $d=1,2,3$ are given by

$$
\begin{aligned}
& g_{m}^{(1)}=-(2 m-3) ! ! /(2 m) ! ! \quad \text { with } g_{1}^{(1)}=-1 / 2 \\
& g_{m}^{(2)}=0 \\
& g_{m}^{(3)}=(2 m-1) ! ! /(2 m) ! !
\end{aligned}
$$

One of the most appealing aspects of Eq. (23) is that the function $F_{n}^{(d)}(\mu)$ contains all of the dimensional and statistical dependence. Moreover, for temperature and particle number ranges where significant deviations from the TF approximation are expected (see Fig. 1), the above sums can be truncated quite quickly. For example, at low temperature and $N=\mathcal{O}\left(10^{3}\right),\left(n_{\max }, k_{\max }, m_{\max }\right)=(50,50,10)$ is more than adequate to obtain single-particle density in any dimension.

Following the $2 \mathrm{D}$ example, it is easy to show that in any dimension, the high-temperature density (i.e., $\left.\mu<\varepsilon_{0}^{(d)}\right)$ is given by

$$
\rho^{(d)}(\mathbf{q} ; T)=2 \sum_{j=1}^{\infty}(-1)^{j+1} e^{j \beta \mu} C_{0}^{(d)}(\mathbf{q} ; j \beta)
$$


In fact, when $\mu<\varepsilon_{0}^{(d)}$, the following identity for fermions exists in all dimensions:

$$
\sum_{j=1}^{\infty}(-1)^{j+1} e^{j \beta \mu}\left(\frac{e^{-j \beta n}+e^{-j \beta(n+1)}}{[2 \sinh (j \beta)]^{d / 2}}\right)=F_{n}^{(d)}(\mu) .
$$

In the $d$-dimensional case, the chemical potential is determined through the normalization condition

$$
\begin{aligned}
N^{(d)} & =\int d^{(d)} q \rho(q, T) \\
& =2 \sum_{n=0}^{\infty}(-1)^{n} F_{n}^{(d)}(\mu) \times F[-n, d / 2 ; 1 ; 2] \\
& =2 \sum_{n=0}^{\infty} \frac{\eta^{(d)}}{\exp \left[\left(\varepsilon_{n}^{(d)}-\mu\right) / T\right]+1},
\end{aligned}
$$

where $F[a, b ; c ; z]$ is a hypergeometric function [18], and $\eta^{(d)}$ denotes the degeneracy of the quantum levels in $d$ dimensions. The functions $F[a, b ; c ; z]$ can only be evaluated analytically for even $d$; their values are given by $(-1)^{n} F[-n, d / 2 ; 1 ; 2]=1,(2 n+1),\left(2 n^{2}+2 n+1\right)$, for $d=2,4,6$, respectively.

\section{B. Single-particle kinetic energy density in $d$ dimensions}

The finite-temperature kinetic energy density can be obtained in a manner entirely analogous to the derivation for the single-particle density. We begin by first investigating two expressions at zero temperature (in scaled units) [3]

$$
\begin{gathered}
\tau(\mathbf{q})=-2 \frac{1}{2} \sum_{\varepsilon_{i}<E_{f}} \phi_{i}^{\star}(\mathbf{r}) \nabla^{2} \phi_{i}(\mathbf{r}), \\
\tau_{1}(\mathbf{q})=2 \frac{1}{2} \sum_{\varepsilon_{i}<E_{f}}\left|\nabla \phi_{i}(\mathbf{r})\right|^{2}
\end{gathered}
$$

where, again, the factor of two accounts for spin. Equations (29), (30) both integrate to the same exact kinetic energy and in the presence of time reversal symmetry are simply related by

$$
\tau(\mathbf{q})=\tau_{1}(\mathbf{q})-\frac{1}{4} \nabla^{2} \rho(\mathbf{q})
$$

At low temperatures, shell effects give rise to oscillations in $\tau(\mathbf{q})$ and $\tau_{1}(\mathbf{q})$ that are exactly opposite in phase [20]. As such, a convenient quantity to consider is their mean:

$$
\begin{aligned}
\xi(\mathbf{q}) & =\frac{1}{2}\left[\tau(\mathbf{q})+\tau_{1}(\mathbf{q})\right] \\
& =-\left.\frac{1}{2} \nabla_{s}^{2} \rho(\mathbf{q}, \mathbf{s})\right|_{s=0} .
\end{aligned}
$$

Starting from Eq. (4) and noting that differentiation commutes with the Laplace inverse operator, we readily obtain after suitable manipulations of hyperbolic functions the following Laplace inverse:

$$
\xi^{(d)}(\mathbf{q} ; T)=\frac{d}{2} \mathcal{L}_{\mu}^{-1}\left[\frac{C_{T}^{(d)}(\mathbf{q} ; \beta)}{\beta} \operatorname{coth}(\beta / 2)\right],
$$

where $C_{T}^{(d)}(\mathbf{q} ; \beta)$ is defined by Eqs. (5, , 7$)$, with $s=0$. This immediately gives

$$
\xi^{(d)}(\mathbf{q} ; T)=\frac{d}{(2 \pi)^{d / 2}} \mathcal{L}_{\mu}^{-1}\left[\frac{1}{4 \sinh ^{2}(\beta / 2)} \sinh ^{(1-d / 2)}(\beta) e^{-q^{2} \tanh (\beta / 2)} \frac{\pi T}{\sin (\pi \beta T)}\right] .
$$


In order to proceed, we substitute Eq. (21) into (34) and make use of the convolution theorem for (two-sided) Laplace transforms just as in Sec. II A. We require the following exact Laplace inverse

$$
\mathcal{L}_{\eta}^{-1}\left[\frac{e^{-n \beta}}{4 \sinh ^{2}(\beta / 2)}\right]=\sum_{k=0}^{\infty}(k+1) \delta(\eta-(2 k+1)-n) \Theta(\eta)
$$

A direct evaluation of Eq. (34) leads to the simple expression for $\xi(\mathbf{q} ; T)$, which is valid in any dimension:

$$
\xi^{(d)}(\mathbf{q} ; T)=\frac{1}{\pi^{d / 2}} \frac{d}{2} \sum_{n=0}^{\infty} G_{n}^{(d)}(\mu)(-1)^{n} L_{n}\left(2 q^{2}\right) e^{-q^{2}}
$$

where

$$
G_{n}^{(d)}(\mu)=\sum_{k=1}^{\infty}(2 k-1)\left[\frac{1}{\exp \left[\left(\varepsilon_{n}^{(d)}+k-1-\mu\right) / T\right]+1}+\sum_{m=1}^{\infty} \frac{g_{m}^{(d)}}{\exp \left[\left(\varepsilon_{n}^{(d)}+k+2 m-1-\mu\right) / T\right]+1}\right]
$$

It is interesting to note that aside from numerical factors, the finite-temperature kinetic energy density and singleparticle density have identical functional forms in all dimensions.

An alternative, and useful formula for the kinetic energy density, $\xi^{(d)}(\mathbf{q} ; T)$, may be obtained from Eq. (33) by noting that

$$
\int_{q}^{\infty} e^{-q^{\prime 2} \tanh (\beta / 2)} q^{\prime} d q^{\prime}=\frac{1}{2} \operatorname{coth}(\beta / 2) e^{-q^{2} \tanh (\beta / 2)}
$$

It then follows that the kinetic energy density is given by

$$
\xi^{(d)}(\mathbf{q} ; T)=\frac{d}{2} \int_{q}^{\infty} \rho^{(d)}\left(\mathbf{q}^{\prime} ; T\right) q^{\prime} d q^{\prime}
$$

\section{Reduction to the TF form for $N \rightarrow \infty$}

At low temperatures (i.e., $T=0$ ) and small particle numbers, the spatial densities, given by Eqs. (23,36), exhibit pronounced shell oscillations, especially in low dimensions [3]. However, as the number of particles is increased, these shell effects get washed out and the spatial densities approach their usual TF form. In this section we show rigorously how the exact $T=0$ density profiles reduce to their TF forms as $N \rightarrow \infty$. For simplicity, we focus only on the $2 \mathrm{D}$ case.

Consider the exact zero-temperature density given by Eq. (16). Putting $2 q^{2}=t$, and using the one-sided Laplace transform

$$
F(s)=\int_{0}^{\infty} \rho^{(2)}(t) \exp (-s t) d t
$$

it is easy to find that

$$
F(s)=\frac{2}{\pi} \sum_{n=0}^{M}(M-n+1) \frac{\left(\frac{1}{2}-s\right)^{n}}{\left(\frac{1}{2}+s\right)^{n+1}} .
$$

The above $n$ sum may be performed exactly [21], yielding

$$
F(s)=\frac{2}{\pi} \frac{1}{8 s^{2}}\left[\left(\frac{1-2 s}{1+2 s}\right)^{M+1}\{1-2 s\}+(4 s M+6 s-1)\right] .
$$

Note that the real part of $s$ must be positive when taking the inverse Laplace transform of $F(s)$. Hence $\left|\frac{2 s-1}{2 s+1}\right|<1$, so that the first term in Eq. (42) vanishes as $M \rightarrow \infty$. This is indeed the case when the number of fermions $N \rightarrow \infty$. In this limit, we get

$$
F(s)=\frac{M}{\pi s}+\frac{3}{2 \pi s}-\frac{1}{4 \pi s^{2}}
$$


Taking the inverse Laplace transform with respect to $t$, we immediately obtain

$$
\rho^{(2)}(t)=\left(\frac{M}{\pi}+\frac{3}{2 \pi}-\frac{t}{4 \pi}\right)=\frac{1}{\pi}\left(M+\frac{3}{2}-\frac{q^{2}}{2}\right) .
$$

The last expression is the large-N asymptotic TF result for the density in $d=2$ dimensions. Its normalization to the correct particle number $N=M^{2}+3 M+2$ for $M+1$ filled shells leads to the Fermi energy $E_{F}=M+3 / 2+O(1 / M)$ in the large-M limit.

An analogous calculation may be performed for the kinetic energy density at $T=0$. Taking $d=2$ in Eq. (36), and going over to the $T=0$ limit, we have

$$
\xi^{(2)}(\mathbf{q} ; T=0)=\frac{1}{\pi} \sum_{n=0}^{M}(-1)^{n}(M-n+1)^{2} L_{n}(t) e^{-t / 2}
$$

As before, we take the one-sided Laplace transform of the above equation with respect to $s$, and perform the $n$ sum exactly. We then take the large $M$ limit, Laplace invert back to the $t=2 q^{2}$ variable and obtain $\left(M+3 / 2-q^{2} / 2\right)^{2} / 2 \pi$, which is just the TF result for the kinetic energy density.

\section{IDEAL BOSE GAS AT FINITE TEMPERATURE}

A powerful aspect of formalism can be illustrated when one considers a trapped ideal Bose gas at finite temperature. In this case, the finite-temperature Bloch density matrix is simply given by (compare with Eq. (5))

$$
C_{T}(\mathbf{q}, \mathbf{s} ; \beta)=-C_{0}(\mathbf{q}, \mathbf{s} ; \beta) \frac{\pi \beta T}{\tan (\pi \beta T)} .
$$

Noting the following inverse Laplace transform

$$
\mathcal{L}_{\mu}^{-1}\left[-\frac{\pi T}{\tan (\pi \beta T)}\right]=\frac{1}{\left[\exp \left(-\frac{\mu}{T}\right)-1\right]}
$$

we observe that Bose statistics serve only to change the behaviour of the functions $F_{n}^{(d)}(\mu)$ and $G_{n}^{(d)}(\mu)$. Explicitly, for the Bose gas we find

$$
\rho^{(d)}(\mathbf{q} ; T)=\left(\frac{1}{\pi}\right)^{d / 2} \sum_{n=0}^{\infty} F_{n}^{(d)}(\mu)(-1)^{n} L_{n}\left(2 q^{2}\right) e^{-q^{2}}
$$

where

$$
F_{n}^{(d)}(\mu)=\sum_{k=0}^{\infty}\left(\frac{1}{\exp \left[\left(\varepsilon_{n}^{(d)}+k-\mu\right) / T\right]-1}+\sum_{m=1}^{\infty} \frac{g_{m}^{(d)}}{\exp \left[\left(\varepsilon_{n}^{(d)}+k+2 m-\mu\right) / T\right]-1}\right)
$$

and similarly,

$$
\xi^{(d)}(\mathbf{q} ; T)=\frac{1}{\pi^{d / 2}} \frac{d}{4} \sum_{n=0}^{\infty} G_{n}^{(d)}(\mu)(-1)^{n} L_{n}\left(2 q^{2}\right) e^{-q^{2}}
$$

where

$$
G_{n}^{(d)}(\mu)=\sum_{k=1}^{\infty}(2 k-1)\left[\frac{1}{\exp \left[\left(\varepsilon_{n}^{(d)}+k-1-\mu\right) / T\right]-1}+\sum_{m=1}^{\infty} \frac{g_{m}^{(d)}}{\exp \left[\left(\varepsilon_{n}^{(d)}+k+2 m-1-\mu\right) / T\right]-1}\right]
$$

Just an in Sec. II, all of the dimensional and statistical dependence is buried in the functions $F_{n}^{(d)}$ and $G_{n}^{(d)}$. Not surprisingly, the Bose versions of $F_{n}^{(d)}(\mu)$ and $G_{n}^{(d)}(\mu)$ differ from their Fermi counterparts only by the sign in front of unity in the denominator. 


\section{Alternative method}

As it happens, there is an alternative method for evaluating the finite-temperature spatial density in the case of Bose statistics which avoids the summations introduced by the inverse Laplace method. One starts by noting that at all temperatures, the Bose distribution function can be expanded as a power series

$$
\frac{1}{e^{\left(\varepsilon_{n}^{(d)}-\mu\right) / T}-1}=\sum_{j=1}^{\infty} e^{j \mu / T} e^{-j \varepsilon_{n}^{(d)} / T}
$$

This immediately allows us to write the finite-temperature density matrix in the form (here, the bosons are taken to be spinless)

$$
\rho^{(d)}\left(\mathbf{r}, \mathbf{r}^{\prime} ; T\right)=\sum_{j=1}^{\infty} e^{j \mu / T} \sum_{n=0}^{\infty} \phi_{n}^{\star}\left(\mathbf{r}^{\prime}\right) \phi_{n}(\mathbf{r}) \exp \left(-j \varepsilon_{n}^{(d)} / T\right) .
$$

Identifying $\beta=1 / T$ in Eq. (3) then allows us to write Eq. (53) as

$$
\rho^{(d)}(\mathbf{q}, \mathbf{s} ; T)=\sum_{j=1}^{\infty} e^{j \beta \mu} C_{0}^{(d)}(\mathbf{q}, \mathbf{s} ; j \beta) .
$$

The finite-temperature particle density is obtained by setting $s=0$ in Eq. (54) and the kinetic energy density is once again evaluated from Eq. (32).

It is straightforward to show that our expressions for the Bose gas obtained by the inverse Laplace method lead to results identical to the so-called alternative method. For example, in the particularly simple case of two dimensions, we have

$$
\rho^{(2)}(\mathbf{q} ; T)=\left(\frac{1}{\pi}\right) \sum_{n=0}^{\infty}(-1)^{n} L_{n}\left(2 q^{2}\right) e^{-q^{2}} \times \sum_{k=0}^{\infty}\left\{\frac{1}{\left[\exp \left(\frac{2 k+n+1-\mu}{T}\right)-1\right]}+\frac{1}{\left[\exp \left(\frac{2 k+n+2-\mu}{T}\right)-1\right]}\right\} .
$$

Expanding the Bose functions as in Eq. (52) and performing the $k$ sums exactly, we obtain $(\beta=1 / T)$

$$
\begin{aligned}
\rho^{(2)}(\mathbf{q} ; T) & =\frac{1}{\pi} \sum_{j=1}^{\infty} \frac{e^{j \beta \mu}}{2 \sinh (j \beta)} \sum_{n=0}^{\infty}(-1)^{n} L_{n}\left(2 q^{2}\right) e^{-q^{2}}\left\{e^{-j n \beta}+e^{-j(n+1) \beta}\right\} \\
& =\sum_{j=1}^{\infty} e^{j \beta \mu} C_{0}(\mathbf{q} ; j \beta)
\end{aligned}
$$

by virtue of Eq. (9). Indeed, in all dimensions and all temperatures, the following identity can be established for bosons

$$
\sum_{j=1}^{\infty} e^{j \beta \mu}\left(\frac{e^{-j \beta n}+e^{-j \beta(n+1)}}{[2 \sinh (j \beta)]^{d / 2}}\right)=F_{n}^{(d)}(\mu)
$$

where $F_{n}^{(d)}(\mu)$ is given by Eq. (49). The analogous result for fermions is given by Eq. (27).

\section{WEAKLY INTERACTING FERMI GAS}

Up to this point, we have been considering noninteracting gases in a trap. In this section we shall investigate (following Ref. [14) a very dilute weakly interacting Fermi gas a finite temperature. We will consider both threedimensional (3D) and 2D geometries and examine the effect of the interaction on the degeneracy of levels in a given HO shell. 


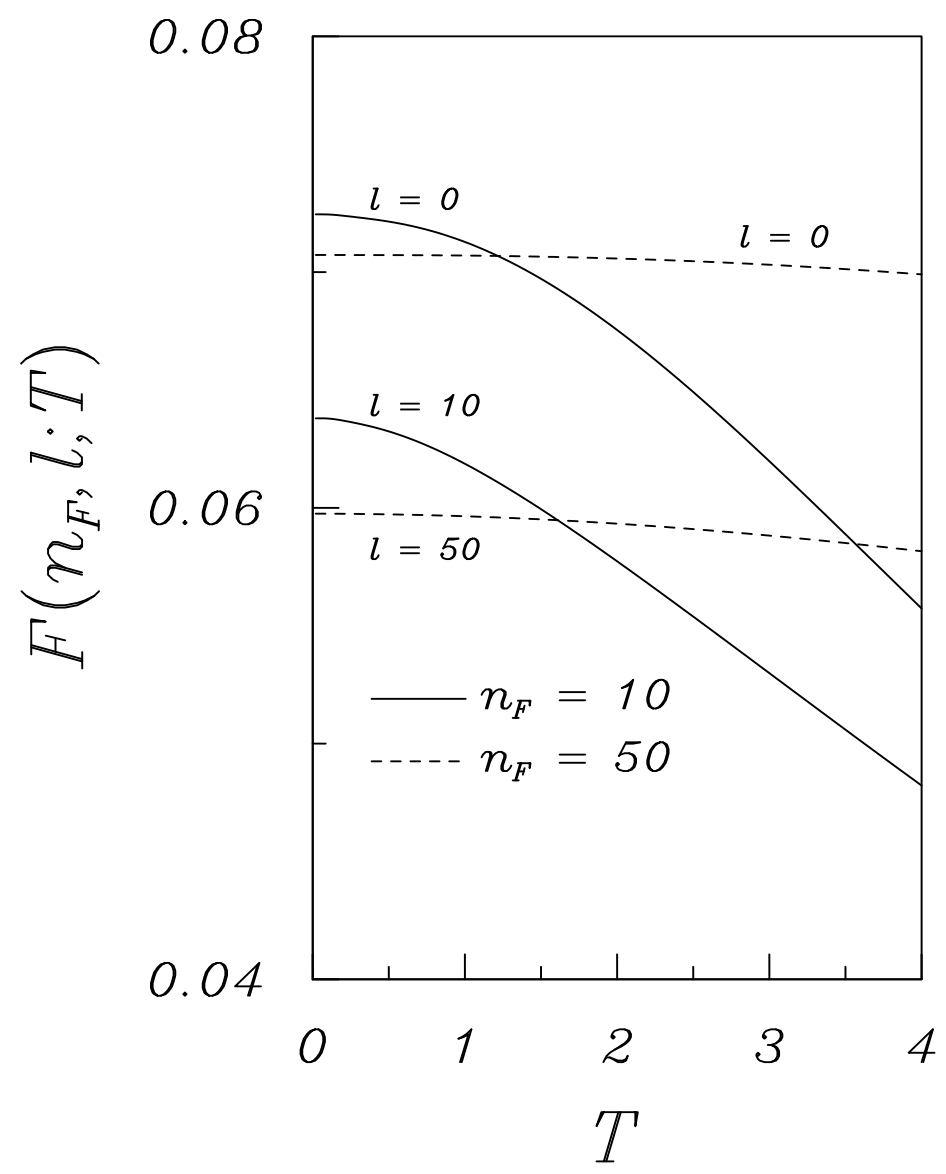

FIG. 2: Finite-temperature energy shift, Eq. (64), for a weakly interacting 3D trapped Fermi gas as a function of the scaled temperature $T$. The solid curves correspond to $n_{F}=10$ and the dashed curves to $n_{F}=50$. Note the weak temperature dependence on the energy shift for $n_{F}=50$.

\section{A. Three dimensions}

We first consider $N$ fermions in a 3D isotropic harmonic oscillator trap interacting via a contact pseudo-potential. The Hamiltonian is then given by

$$
H=\sum_{i=1}^{N}\left(\frac{\mathbf{p}_{i}^{2}}{2}+\frac{1}{2} \mathbf{r}_{i}^{2}\right)+4 \pi a \sum_{i<j} \delta^{3}\left(\mathbf{r}_{i}-\mathbf{r}_{j}\right) .
$$

In the above, $a$ is the dimensionless s-wave scattering length. The mean-field potential at temperature $T$ is

$$
U(r ; T)=2 \pi a \rho(r ; T),
$$

where, keeping in mind that $d=3$, we omit the superscripts on all quantities. If the system is very dilute in the sense that $\hbar \omega \gg|U|$, we may use the unperturbed expressions, Eq. (23), for $\rho(r ; T)$ to obtain $U(r ; T)$. To fix the notation for the degenerate states in a shell, we consider the noninteracting system at $T=0$, for which the Fermi energy is

$$
E_{F}=\left(n_{F}+3 / 2\right),
$$

and $n_{F}$ is the principal HO quantum number at the Fermi surface. The quantum number $n_{F}$ is related to the number of fermions, $N$, in the well by

$$
N=\frac{1}{3}\left(n_{F}+1\right)\left(n_{F}+2\right)\left(n_{F}+3\right) .
$$




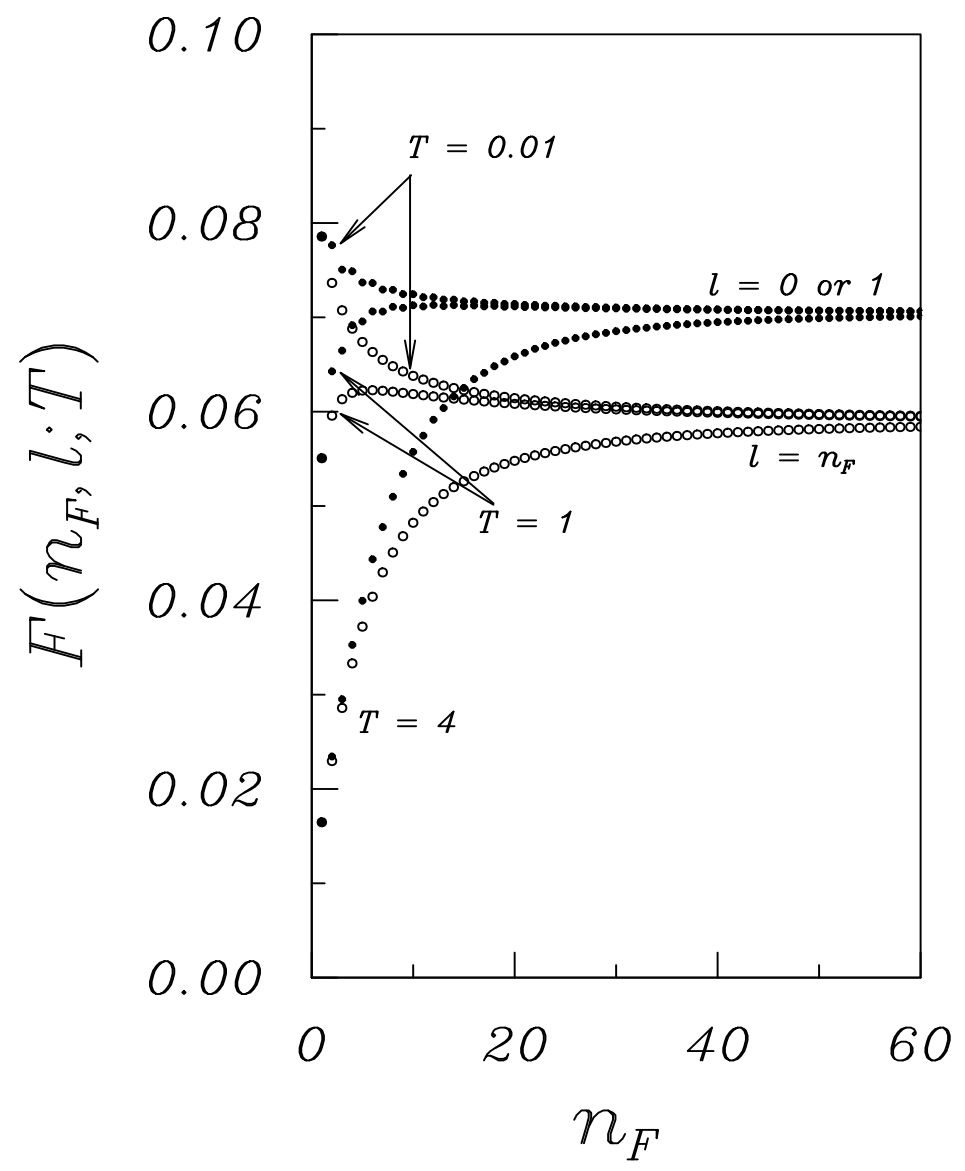

FIG. 3: Finite-temperature energy shift, Eq. 64), for the 3D weakly interacting Fermi gas as a function of $n_{F}$. The open circles correspond to $l=n_{F}$ whereas the filled circles are for $l=0$ or $l=1$. For large $n_{F}, F\left(n_{F}, l ; T\right)$ approaches a constant value, as is expected in the TF limit.

Note that the shell specified by $n_{F}$ has degenerate states with angular momenta $l=n_{F}, n_{F}-2, n_{F}-4, \ldots ., 1$ or 0 . Consider now a particular radial HO state $\mathcal{R}_{n l}$, with $(2 n+l)=n_{F}$ in this shell. Due to the mean-field potential $U(r ; T)$, the single-particle energy of this state is shifted (in lowest order perturbation theory) by the amount [14]

$$
\epsilon_{n_{F}, l}-E_{F}=\Delta \epsilon_{n_{F} l}(T)=\int U(r ; T)\left|\mathcal{R}_{n l}\right|^{2} r^{2} d r .
$$

Thus the degeneracy of the various angular momentum states is lifted by the temperature-dependent interaction (59). Using Eqs. (59) and (23), we note that the relevant radial integral to obtain the energy shift is of the form (at any temperature $T$ )

$$
I=\int_{0}^{\infty} r^{2 l+2} e^{-2 r^{2}} L_{m}\left(2 r^{2}\right)\left[L_{n}^{l+1 / 2}\left(r^{2}\right)\right]^{2} d r .
$$

Although it is possible to obtain an analytical expression for the above integral, in practice it is more convenient to calculate it numerically. In Fig. 2, we display the results for the energy shift as a function of the scaled temperature $T$ for $l=0$ and $l=n_{F}$, with $n_{F}=10$ and 50. Since this energy shift obviously depends on the scattering length $a$ and $n_{F}$, we choose to plot the dimensionless quantity

$$
F\left(n_{F}, l ; T\right)=\frac{\Delta \epsilon_{n_{F} l}(T)}{2 \pi a N^{1 / 2}} .
$$

Our definition of $F\left(n_{F}, l ; T\right)$ in Eq. (64) is a little different from that in [14], where the calculation was performed at $T=0$ in the TF approximation. Note that the temperature dependence for $n_{F}=10$ is much more pronounced than 
for $n_{F}=50$. This would be less so if one scaled the temperature in units of the Fermi energy rather than the oscillator spacing. We also show in Fig. 3 the level splittings, $F\left(n_{F}, l ; T\right)$, as a function of $n_{F}$ at various temperatures. For large $n_{F}$, we observe that $F\left(n_{F}, l ; T\right)$ approaches a constant, demonstrating that $\Delta \epsilon_{n_{F} l}(T)$ behaves as $N^{1 / 2} \simeq n_{F}^{3 / 2}$, as is to be expected in the TF limit.

\section{B. Two dimensions}

We now calculate the corresponding energy shift in the quasi-2D harmonic oscillator trap. A highly anisotropic 3D oscillator will behave as such when the oscillator frequency in one direction (say $\omega_{z}$ ) is much, much greater than in the other two $\left(\omega_{x}=\omega_{y}=\omega_{\perp}\right)$. Furthermore, the temperature $T$ under consideration is low enough so that $k_{B} T \ll \hbar \omega_{z}$. If the interatomic interaction is so weak that it cannot cause excitations in the $z$ direction, we may restrict the Hilbert space in the $z$ direction by setting the oscillator quantum number $n_{z}=0$. The effective interaction of the quasi-2D system is then obtained by taking the 3D delta-function potential in Eq. (58), and taking its expectation value with respect to the $\mathrm{HO}$ wave function in the $z$ direction,

$$
\phi_{0}(z)=\left(\frac{\omega_{z}}{\pi}\right)^{1 / 4} \exp \left(-\omega_{z} z^{2} / 2\right) .
$$

The resulting quasi-2D Hamiltonian is then given by (again, scaled units are used)

$$
H=\sum_{i=1}^{N}\left(\frac{\mathbf{p}_{i}^{2}}{2}+\frac{1}{2} \mathbf{r}_{i}^{2}\right)+g \sum_{i<j} \delta^{2}\left(\mathbf{r}_{i}-\mathbf{r}_{j}\right)
$$

where the momenta and coordinates are planar vectors, $g=2 \sqrt{2 \pi}\left(a / \ell_{z}\right)$ is the effective $2 \mathrm{D}$ dimensionless coupling constant, and we have denoted the oscillator length in the $z$ direction by $\ell_{z}$. We restrict this calculation to $T=0$, although the extension to finite temperature is straightforward. The mean-field one-body potential is then given by

$$
U^{(2)}(r)=\frac{g}{2} \rho^{(2)}(r)
$$

where the $2 \mathrm{D} \rho^{(2)}(r)$ is given by Eq. (16). Identifying $M$ with $n_{F}$, we note that for the $2 \mathrm{D}$ case,

$$
N=\left(n_{F}+1\right)\left(n_{F}+2\right), \quad E_{F}=\left(n_{F}+1\right) .
$$

The radial 2D HO wavefunction is given by

$$
R_{n l}(r)=\left[\frac{2 n !}{(n+|l|) !}\right]^{1 / 2} r^{|l|} e^{-r^{2} / 2} L_{n}^{|l|}\left(r^{2}\right)
$$

with the normalization $\int_{0}^{\infty} d r r\left|R_{n l}\right|^{2}=1$. The energy shift analogous to Eq. 62) is

$$
\Delta \epsilon_{n_{F}, l}^{(2)}=\int_{0}^{\infty} d r r U(r)\left|R_{n l}\right|^{2}=\sqrt{2 \pi}\left(\frac{a}{\ell_{z}}\right) \int_{0}^{\infty} d r r \rho(r)\left|R_{n l}\right|^{2},
$$

and

$$
F^{(2)}\left(n_{F}, l\right) \equiv \frac{\Delta \epsilon_{n_{F}, l}^{(2)}}{\sqrt{2 \pi}\left(\frac{a}{\ell_{z}}\right) N^{1 / 2}} .
$$

The $2 \mathrm{D}$ energy shift at $T=0$ is shown in Fig. 目. It readily seen from this figure that $F^{(2)}\left(n_{F}, l\right)$ depends on $n_{F}$, but not explicitly on $l$. Consequently, unlike the $3 \mathrm{D}$ case, there is no splitting (in the lowest order perturbation theory) between states with different $l$ values in a given shell at $T=0$. This surprising result may be also checked analytically. Specifically, a little algebra gives

$$
\Delta \epsilon_{n_{F}, l}^{(2)}=2 \sqrt{\frac{2}{\pi}} g \frac{n !}{(n+|l|) !} \sum_{m=0}^{n_{F}}(-1)^{m}\left(n_{F}-m+1\right) \int_{0}^{\infty} d x x^{|l|} e^{-2 x} L_{m}(2 x)\left[L_{n}^{|l|}(x)\right]^{2} .
$$




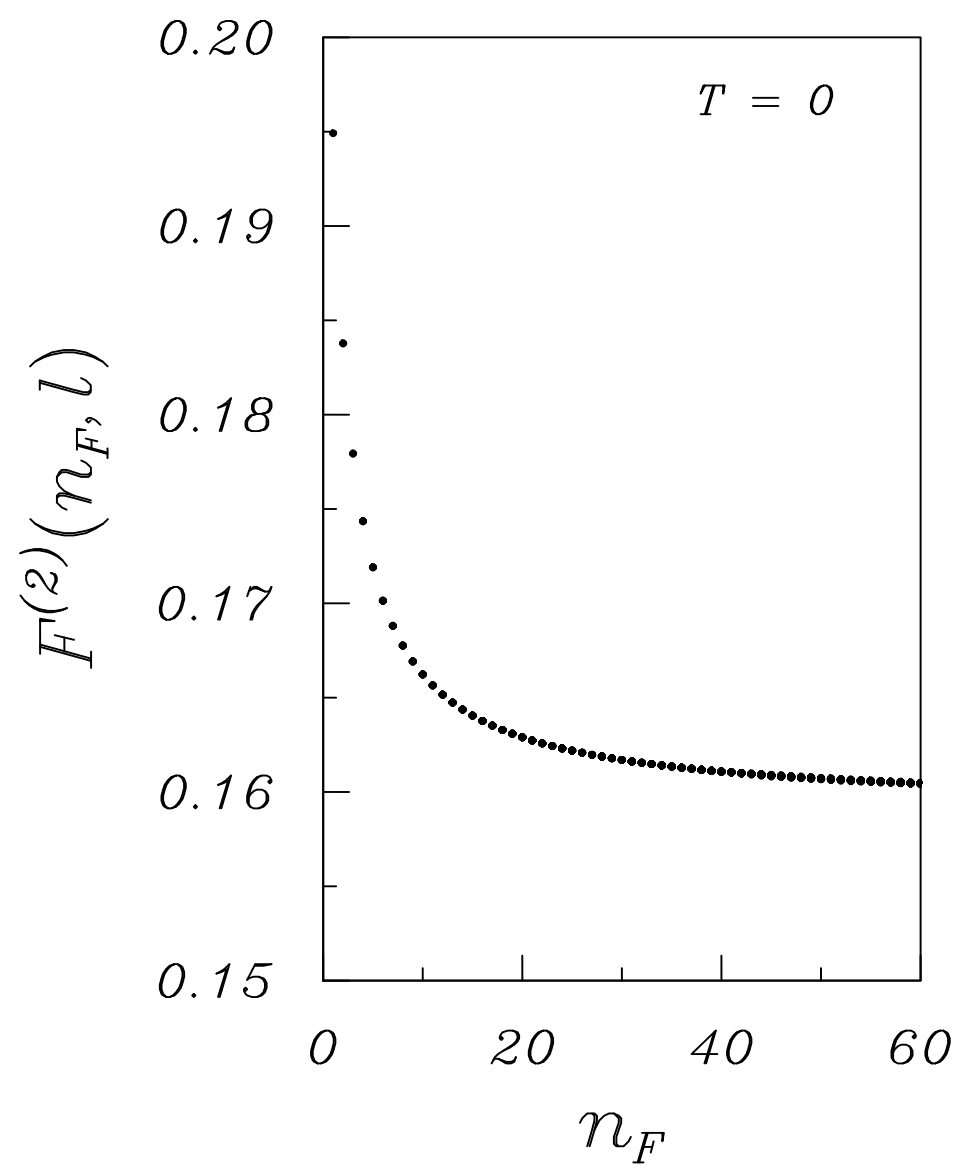

FIG. 4: The energy splitting $F^{(2)}\left(n_{F}, l\right)$ defined by Eq. $(71)$ for a 2 D Fermi gas at $T=0$. Note that $F^{(2)}\left(n_{F}, l\right)$ is independent of $l$ for every HO shell.

The integral above can be performed exactly by noting that 18

$$
\left[L_{n}^{|l|}(x)\right]^{2}=\frac{(n+|l|) !}{n !} \sum_{k=0}^{n} \frac{x^{2 k}}{(|l|+k) ! k !} L_{n-k}^{|l|+2 k}(2 x) .
$$

The final result is given by (with the restrictions $(n-k) \leq m \leq(n+k+|l|))$

$$
\Delta \epsilon_{n_{F}, l}^{(2)}=2 \sqrt{\frac{1}{2 \pi}} g \sum_{m=0}^{n_{F}}\left(n_{F}-m+1\right) \sum_{k=0}^{n} \frac{(-1)^{n-k}}{2^{|l|+2 k}} \frac{(|l|+2 k) !(n+k+|l|) !}{k !(n-k) !(|l|+k) !(m-n+k) !(n+k+|l|-m) !} .
$$

An explicit case by case evaluation of the above expression [21] verifies that the shift depends only on $n_{F}$, and is independent of $|l|$; we have not been able to demonstrate this fact analytically for a general $n_{F}$. As stated earlier, the independence of $\Delta \epsilon_{n_{F}, l}^{(2)}$ on $|l|$ is to be expected for large $n_{F}$ in which the TF density depends only on $r^{2}$. What is surprising is that the shift is independent of $l$ for all $l$ within a given shell even though the exact single-particle density, Eq. (16), has been used.

\section{SUMMARY AND DISCUSSION}

We have presented for the first time exact analytical expressions for the number and kinetic energy densities of an ideal Fermi gas in a harmonic trap at finite temperatures. These new expressions, given by Eq. (23) and (36), are 
valid at any temperature, and in any dimension. We have also derived the corresponding results for bosons, which are given by Eqs. (48) and (50). Unlike previous expressions in the literature (see e.g., Ref. [10]), our functional forms for the particle and kinetic energy densities clearly illustrate the role of the quantum statistics and have a universal form independent of the dimension of the system. We have also demonstrated analytically in Sect. II C (for $d=2$ ), how the exact fermionic expressions for the particle and kinetic energy densities at $T=0$ reduce to their TF forms as $N \rightarrow \infty$. It is worthwhile noting that establishing this reduction analytically using a wavefunction representation for the particle and kinetic energy densities is a non-trivial task (see e.g., Ref. [9]).

To complement our ideal gas results, we have also considered a weakly interacting trapped Fermi gas. The exact fermionic expression for the spatial density was used to obtain the mean-field potential, and the finite temperature first-order shifts in the energy levels for a given HO shell. Similar calculations were recently reported by Heiselberg and Mottelson [14 in 3D, but only at $T=0$ and within the TF approximation. As was pointed out in Ref. [14, for attractive interparticle interactions (i.e., negative $a$ ), there are also pairing correlations between time-reversed states $(l, m)$ and $(l,-m)$ in a given shell. In the situation when the splittings given by Eq. (62) are small compared with the pairing gap, the pairing may take place between all states of a shell leading to supergaps. We plan on performing a detailed study of the supergap phenomenon at finite temperatures in a future publication.

As our final application, we also studied the energy level splittings in a $d=2$ isotropic HO geometry at $T=0$. We found the surprising result that the $l$-degeneracy for a given HO shell is not lifted in the lowest order perturbation theory, even when the exact expression for the single-particle density is used. In the $2 \mathrm{D}$ TF approximation, this result

can be understood by noting that the TF particle density, $\rho_{\mathrm{TF}}^{(2)}(r) \propto\left(\mu-r^{2} / 2\right)$, does not break the $S U(2)$ symmetry of the harmonic trap. However, the radial dependence for the exact density (especially for a small number of fermions) is more complicated, and therefore our result is unexpected. Given this finding, it should prove very interesting to study the nature of pairing transitions in a 2D trapped Fermi system at finite temperature.

\section{Acknowledgments}

This work was supported in part by the National Sciences and the Engineering Research Council of Canada (NSERC) and by the Canadian Institute for Advanced Research (CIAR). MB acknowledges travel support by the Deutsche Forschungsgemeinschaft. BVZ would like to thank Dr. J. P. Carbotte for additional financial support.

[1] F. Dalfovo, S. Giorgini, L. P. Pitaevskii, and S. Stringari, Rev. Mod. Phys. 71, 463 (1999).

[2] B. DeMarco and D. S. Jin, Science 285, 1703 (1999); M. J. Holland, B. DeMarco, and D. S. Jin, Phys. Rev. A 61, 053610 (2000).

[3] M. Brack and B. P. van Zyl, Phys. Rev. Lett. 86, 1574 (2001).

[4] J. Schneider and H. Wallis, Phys. Rev. A 57, 1253 (1998).

[5] F. Brosens, J. T. Devreese, and L. F. Lemmens, Phys. Rev. E 57, 3871 (1998).

[6] P. Vignolo, A. Minguzzi, and M. P. Tosi, Phys. Rev. Lett. 85, 2850 (2000).

[7] Z. Akdeniz, P. Vignolo, A. Minguzzi, and M. P. Tosi, cond-mat/0205480 v1 (2002).

[8] G. M. Bruun and C. W. Clark, Phys. Rev. A 61, 061601 (2000).

[9] F. Gleisberg, W. Wonneberger, U. Schlöder, and C. Zimmermann, Phys. Rev. A 62, 063602 (2000).

[10] X. Z. Wang, Phys. Rev. A 65, 045601 (2002).

[11] D. A. Butts and D. S. Rokhsar, Phys. Rev. A 55, 4346 (1997).

[12] Specifically, the results for fermions in Ref. [10] are only valid for temperatures where $\mu<\varepsilon_{0}$ (see Sec. IIA).

[13] E. H. Lieb, Rev. Mod. Phys. 53, 603 (1981); 54, 311 (1982) erratum.

[14] H. Heiselberg and B. Mottelson, Phys. Rev. Lett. 88,190401 (2002).

15] B. van der Pol and H. Bremmer, Operational Calculus, (Cambridge University Press, Cambridge, U.K.), Second Ed. (1955).

[16] M. Brack and R. K. Bhaduri, Semiclassical Physics, Addison-Wesley, part of the Frontiers in Physics vol. 96 (1997).

[17] E. H. Sondheimer and A. H. Wilson, Proc. R. Soc. London A 210, 173 (1951).

[18] I. S. Gradshteyn and I. M. Ryzhik, Table of Integrals, Series, and Products (Academic Press, New York, 1994), 4th ed., Eq. 8.975.1. Also see Eq. (8.976.4). Note that there is a misprint in the latter formula; the upper limit in the sum is not infinity, but $n$.

[19] In two dimensions, the finite-temperature TF density is given by $\rho_{\mathrm{TF}}^{(2)}(r ; T)=(T / \pi) \ln (1+\exp [\beta(\mu-V(r))])$.

[20] R. K. Bhaduri and L. F. Zaifman, Can. J. Phys. 57, 1990(1979); C. Guet and M. Brack, Z. Phys. A 297, 247 (1980).

[21] We have used the mathematical software package, Maple ${ }^{(}$, to evaluate this quantity. 